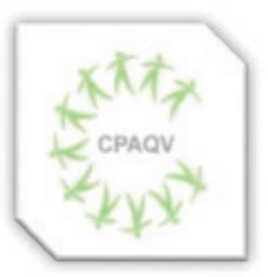

ISSN: 2178-7514

Vol. 14| No. 1| Ano 2022
ARTIGO DE REVISÃO

\section{PROMOÇÃO DA SAÚDE COM EXERCÍCIO FÍSICO PARA PESSOAS COM DIABETES: UMA REVISÃO NARRATIVA}

\section{Health promotion with physical exercise for people with diabetes: a narrative review}

Pedro P. Abdalla ${ }^{1,5}$; Guanis B. Vilela Junior ${ }^{1}$; Adriano A. Pereira ${ }^{1}$; Alexandre F. Carvalho ${ }^{13}$; Anderson Martelli; Bráulio N. Lima ${ }^{1}$; Gustavo C. Martins ${ }^{1,9,10,11}$; Heleise F. R. Oliveira ${ }^{1,14}$; José Ricardo L. Oliveira ${ }^{1}$; Klebson S. Almeida ${ }^{1,8}$; Luis F. Silio ${ }^{1}$; Marcelo F. Rodrigues ${ }^{1}$; Mariela S. Maneschy ${ }^{8}$; Uebister I. S Guedes ${ }^{7}$; Paulo M. B. Cavaco ${ }^{12}$; Anderson S. Carvalho ${ }^{1,6}$; Ricardo P. Passos ${ }^{1,2}$

\title{
RESUMO
}

A Diabetes Mellitus é uma doença crônica frequente no mundo, no qual o número de vítimas de suas complicações associadas vem aumentando. Isso ocorre devido ao sedentarismo da população, que tem quadro geral de saúde agravado devido também aos maus hábitos alimentares. Muito se discute sobre o papel do exercício físico na rotina e na saúde da população portadora da doença, pois muitas vezes o exercício é considerado benéfico para a saúde física e mental, podendo ser uma medida terapêutica preventiva ou paliativa desta doença. O papel da gestão deste exercício pelo profissional responsável é importante, pois é através dele que se permite a melhora do metabolismo. Neste ponto foi discutido que a promoção de saúde para a população, não somente portadora da doença, pode expandir a visão em um contexto geral, possibilitando desenvolvimento humano, dando visibilidade para um envelhecimento saudável. Este trabalho enfatiza a possibilidade de utilizar a tecnologia para divulgar e motivar a realização de exercício físico para minimizar a incidência de diabetes na população. $\mathrm{O}$ método utilizado foi uma revisão narrativa da literatura. Conclui-se: pessoas sedentárias com hábitos alimentares inadequados têm maiores chances de adquirir a diabetes e ter de fazer uso de medicamentos para controlar a doença. Utilizar o exercício físico como medida terapêutica pode auxiliar no tratamento e prevenção da diabetes.

Palavras-chave: Atividade; Física; Melhora; Patologia;

\begin{abstract}
Diabetes Mellitus is a frequent chronic disease in the world, in which the number of victims of its associated complications is increasing. This is due to the sedentary lifestyle of the population, whose general health condition is worsened due to poor eating habits. Much is discussed about the role of physical exercise in the routine and health of the population with the disease, as exercise is often considered beneficial for physical and mental health, and can be a preventive or palliative therapeutic measure for this disease. The role of the management of this exercise by the responsible professional is important because it is through it that the improvement of metabolism is allowed. At this point, it was discussed that health promotion for the population, not only those with the disease, can expand the vision in a general context, enabling human development, giving visibility to healthy aging. This work emphasizes the possibility of using technology to promote and motivate physical exercise to minimize the incidence of diabetes in the population. The method used was a narrative review of the literature. It is concluded: sedentary people with inadequate eating habits are more likely to acquire diabetes and have to use medication to control the disease. Using physical exercise as a therapeutic measure can help in the treatment and prevention of diabetes.
\end{abstract}

Keywords: Activity; Physical; Improvement; Pathology.

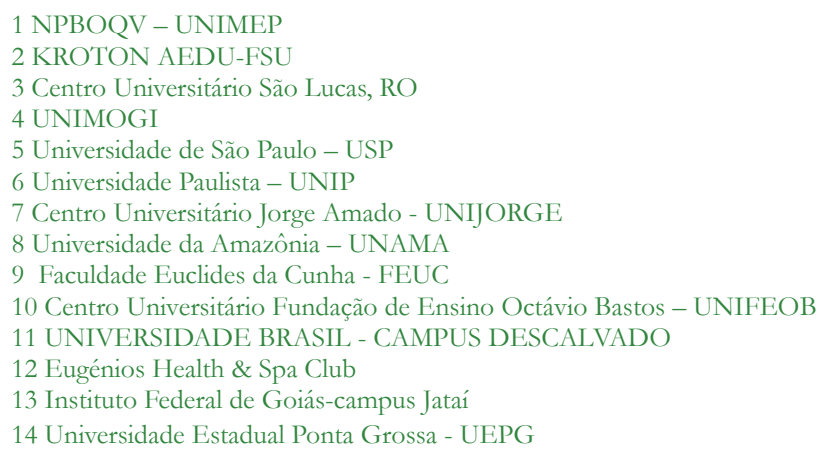

Autor de correspondência

Ricardo Pablo Passos - rppasso@gmail.com

DOI: $10.36692 / \mathrm{v} 14 \mathrm{n} 1-11 \mathrm{R}$ 


\section{INTRODUÇÃO}

Mesmo diante do avanço tecnológico e a globalização, que permitem um conhecimento maior sobre hábitos que não são saudáveis para o ser humano, presencia-se um cenário de aumento de casos de diabetes tipo II na população. Por isto o investimento na qualidade de vida através da tecnologia poderia contribuir para o desenvolvimento sustentável da população e minimizar a prevalência desta doença. Segundo pesquisas realizadas pelo IBGE (1) existem aproximadamente 14 milhões de pessoas no Brasil que são diagnosticadas com diabetes do tipo II e até o ano de 2035 este número tem a possibilidade de ser mais que dobrado, passando a 30 milhões de pessoas com a doença no país.

Tem sido um desafio ter a massiva presença da população em prática de atividades físicas consideráveis. Uma das explicações para estas situações é acesso a tecnologias para todos os públicos, mas que ainda não é bem utilizada nesse sentido. Icentivar a prática de exercícios físicos deveria ser constante através dessas mídias, pois permitiria um envelhecimento saudável com menos problemas de saúde.

Portanto, nos perguntamos se seria possível expor à população como é possível utilizar a tecnologia para divulgar e motivar a realização de exercícios físicos com a expectativa de diminuir e minimizar a incidência de diabetes?
Desta forma, este trabalho teve como objetivo compreender a importância do exercício físico como subsídio para a melhoria na qualidade de vida para pessoas com diabetes, caracterizando o impacto do avanço tecnológico na vida de pessoas com diabetes.

\section{MÉTODOS}

Este trabalho foi pesquisa revisão bibliográfica narrativa e qualitativa, fundamentada em livros, artigos científicos, teses e dissertações. Foram utilizadas as bases de dados Google acadêmico e Scielo, sem restrições quanto ao período de pulibacção, no idioma português e com as palavras chaves: Atividade Física, Exercício Físico, Diabetes, Patologia. Nos dividimos esse estudo em três tópicos principais: 1) impactos dos avanços tecnológicos na diabetes; 2) influência do sedentarismo e habitos alimentares na vida de pessoas com diabetes; 3) benefícios da atividade fisica na saude de pessoas com diabetes.

\section{IMPACTOS DOS AVANÇOS TECNOLÓGICOS NA DIABETES}

A Diabetes Mellitus tipo II é uma das doenças crônicas mais frequentes em todo o mundo (2). O número de casos causados pela diabetes entre os anos de 1995 e 2025 aumentará em 122\% em todo os países do mundo (3). Para a Sociedade Brasileira de Endocrinologia e Metabologia, a Diabete Mellitus é uma doença 
que se diagnostica devido a elevação da glicemia no sangue e pode ser causada pela deficiência na secreção da insulina, um hormônio produzido pelo pâncreas. A insulina estimula o mecanismo para remover do sangue a quantidade elevada de glicose e também de fornecer a entrada em outras células do corpo humano. Quando este processo não funciona corretamente, ocorre um acúmulo de glicose no sangue, no qual é chamado como hiperglicemia. A sociedade Brasileira de Endocrinologia e Metabologia ressalta que hiperglicemia, quando não tratada pode ocasionar diversas lesões na circulação sanguínea, prejudicando assim o desempenho de órgãos importantes, como por exemplo os rins, coração e nervos. Por isto quanto antes o diagnóstico da doença acontecer, melhor será para o indivíduo. A diabetes, é basicamente dividida em dois tipos. O tipo I, no qual a doença se manifesta em pessoas que possuem o sistema imunológico desordenado, que destrói suas próprias células betas do pâncreas. Esta variação é mais incidente em crianças e jovens, e esses precisam realizar a aplicação de insulina para controlar a glicemia. Já o tipo II se manifesta quando o corpo não consegue fazer uso da insulina de forma satisfatória. Então, o pâncreas ainda realiza a produção de insulina normalmente, ou não produz a quantidade suficiente. Nos dois casos é verificado um aumento de glicose no sangue, sendo esse tipo II mais comum em adultos e idosos, no qual em níveis iniciais pode ser tratado com uma alimentação saudável, exercícios físicos e medicamentos orais.

Houve um aumento na expectativa de vida da população do mundo, porém existem alguns hábitos, como por exemplo, a diminuição de práticas de exercícios físicos, novas tecnologias, obesidade, etc., que contribuem para o crescimento da incidência da diabetes (4). O mesmo autor afirma que o sobrepeso e a obesidade são dois fatores que influenciam diretamente como um risco para a obtenção da diabetes tipo II, além de poder estar ligada diretamente ao sedentarismo, os avanços de tecnologias mostram também que problemas genéticos podem favorecer a resistência a insulina, aumentando as chances de diabetes. A tecnologia vem mudando e se transformando todos os dias, ganhando cada vez mais espaço no cotidiano infantil. As atividades físicas e afazeres tradicionais disputam atenção com as mídias, redes sociais e sedentarismos no qual influenciam diretamente e negativamente na incidência de diabetes. Mais de 150 mil adultos em cerca de 17 países. possuem em casa aparelhos eletrônicos como por exemplo televisão e computador (5). Além disto, estas mesmas pessoas, preferem utilizar o carro como meio oficial de locomoção. Para o mesmo autor, eles estão mais propensos à obesidade e ao diabetes tipo II. A taxa das duas doenças é de 3,4\% nos indivíduos que não possuem os aparelhos tecnológicos, e nos que possuem é muito maior: $14,5 \%$ para obesidade 
e $11,7 \%$ para diabetes. Para Cerezo (6) o aumento da diabetes tipo II aumentou com o avanço da globalização e com a divulgação de novas tecnologias no decorrer dos anos, cada vez estão mais acessíveis para a população, na qual deixam de praticar atividades físicas. Para Previtale (7), os indivíduos da sociedade moderna se isolam dentro de suas casas com suas tecnologias e passam a deixar de expressar seus sentimentos publicamente, o que segundo o autor é ruim para o desenvolvimento cognitivo e físico do indivíduo. A utilização da tecnologia na vida das pessoas em geral se apresenta cedo, seja pelo aparelho de televisão, celular, etc o que levou pensadores a levantar questões acerca do desenvolvimento de uma criança e sua saúde que substituem cada vez mais suas amizades e brincadeiras reais por jogos e amizades virtuais. E com isto tem aumentando a incidência da diabetes tipo II quando adultos. Hanauer (8) afirma que a tecnologia vem substituindo alguns dos hábitos saudáveis que envolvem atividades físicas que para a sociedade de gerações passadas eram tradicionais. Assim mesmo que de forma silenciosa, muitas pessoas deixam de sair de casa, pois possuem tudo que precisam em seus computadores. O mesmo autor discute o quanto isso pode ser ruim para as relações interpessoais e até mesmo para a saúde de um determinado individuo, uma vez que ao deixar de sair de casa, ele deixa de praticar exercícios físicos. Isso seria um ponto importante para a manutenção da saúde e permite que a incidência da diabetes tipo II seja evitada, uma vez que com o aumento do gasto energético há diversos benefícios, como por exemplo, o controle da insulina e sua sensibilidade no sangue. As pessoas utilizam toda esta tecnologia e as inovações que os deixam cada vez mais dependentes. Elas possuem seus dispositivos eletrônicos para acesso a qualquer horário do dia, o que leva a uma preocupação de como esta presencialidade pode ser prejudicial a este grupo. Segundo Velloso (9), a tecnologia e a internet não possuem um limite ou restrição para seus usuários. Principalmente crianças e adolescentes que passam por uma fase de transição tão importante de desenvolvimento corporal, hormonal e intelectual, influenciando negativamente para que estes fiquem com problemas futuros. Por este motivo a prioridade para as gerações futuras deveria ser de pelo menos realizar uma atividade física no dia que proporcione um desenvolvimento e crescimento saudável, com a finalidade de implantar metodologias que proporcionem uma melhor qualidade de vida. Seria importante realizar informativos sobre as consequências da diabetes (complicações nos rins, coração, artérias e etc) e de como o exercício físico pode ser um importante instrumento no combate para esta doença.

Mas a tecnologia também poderia contribuir para o tratamento e prevenção da diabetes quando bem utilizada, como explica 
a Sociedade Brasileira de Diabetes. A questão de uso da tecnologia para os indivíduos para o combate e prevenção a doenças é um processo que ainda está engatinhando, pois ainda existem diversas ações que buscam garantir a aprendizagem e transformação na vida da sociedade ao que se refere a área de saúde. O Ministério da Saúde (10) adotou diversas estratégias direcionadas a levar diversas informações a população e profissionais da saúde quanto ao benefício do uso da tecnologia para combate a doenças. Com isto, buscaram fortalecer e incentivar a prática de exercícios físicos. Por meio de um programa de incentivo a conscientização de uso de tecnologias em prol da saúde, a população poderá se beneficiar de diversas descobertas científicas, que por sua vez será capaz de auxiliar no combate e diagnóstico simplificado de doenças. A tecnologia vem revolucionando o tratamento de diabéticos e proporciona ferramentas que quando frequentemente aplicadas, são essenciais para o tratamento desta doença que ainda não possui cura, mas que contribuem para um equilíbrio na glicemia.

\section{INLUÊNCIA} SEDENTARISMO E HABITOS ALIMENTARES NA VIDA DE PESSOAS
COM DIABETES

O sedentarismo está associado com mudanças nos hábitos alimentares, além disso caracteriza pessoas com menos frequência de atividades físicas, sendo considerado um dos maiores problemas da saúde pública. É caracterizado quando as atividades sem gasto energético significativo, como assistir televisão sentado/deitado, utilizar o smartphone e jogar vídeo game somente com movimentos de habilidade motora fina em períodos prolongados, onde o esforço físico realizado é baixo ou nulo. O sedentarismo envolve risco para todos os seres humanos, pois ele possibilita a degradação do sistema imunológico, permitindo também o aparecimento de doenças crónicodegenerativas não transmissíveis, como por exemplo, a diabetes tipo II, câncer, obesidade, hipertensão e doenças cardiovasculares, sendo estas são responsáveis pelo maior índice de mortalidade e incapacidades nos seres humanos. O conhecimento da população sobre os dados de sedentarismo e seus agravantes na saúde contribui para a melhora da saúde pública, pois pode ser um subsídio para o melhor incentivo e gerenciamento das práticas as atividades físicas (11). O percentual de pessoas que são sedentárias no período de 365 dias na população de 15 anos ou mais em 2015, segundo o IBGE, variou entre 36,3 e 41,1\% nas diferentes regiões do Brasil. Portanto, grande parte da população não realiza atividades físicas suficientes.

A promoção de qualidade de vida está diretamente relacionada ao estilo de vida do indivíduo. Quando o ser humano investe em sua saúde e pratica exercícios abrindo mão do sedentarismo, o mesmo está investindo para 
uma melhora na saúde pública e diminuição das doenças causadas pelo sedentarismo. A falta de atividade física é um problema moderno causada por hábitos não saudáveis e de alimentação incorreta. O sedentarismo, quando persiste por longos períodos na vida do indivíduo, podee acarretar diversas doenças e problemas de saúde, causando limitações a longo prazo. Segundo Zlochevsky (12) a obesidade é um distúrbio metabólico causado principalmente por falta de exercícios físicos e maus hábitos alimentares. A obesidade pode aumentar a reincidência para casos de diabetes tipo II, além de outros diversos problemas de saúde. Segundo o mesmo autor, os principais causadores da diabetes é o sobrepeso e o sedentarismo, no qual um influencia diretamente um ao outro. $\mathrm{O}$ autor informa que quanto mais massa corporal o individuo tiver maior é o risco de desenvolvimento da diabetes. Pessoas que possuem sobrepeso e são sedentárias possuem a necessidade de um acompanhamento médico, no qual orientaria ações para o controle metabólico, estimulando uma alimentação saudável e exercícios físicos específicos. Os maus hábitos adotados pelo individuo durante sua infância e adolescência poderão se refletir quando adultos, uma vez que a diabete raramente se manifesta nestas idades (exceto o tipo I). Segundo pesquisas divulgadas pela Abeso em 2011 (13), no Brasil entre 2002 e 2005 cerca de 737.667 pessoas possuíam a diabetes, sendo que deste valor total, cerca de 363.881 não realizavam nenhum tipo de exercício físico e/ou mantinham uma alimentação saudável. Desta forma, foi considerado que cerca de $49,3 \%$ da população portadora da diabetes é sedentária. Desta forma, é preciso realizar incentivos para obtenção de hábitos alimentares saudáveis e a práticas de exercícios físicos como uma forma de combater alguns agravantes do mau estado de saúde causados pelos hábitos sedentários. Uma vez que o aumento da adiposidade dificulta a ação da insulina no organismo, prevenir os casos de sobrepeso e obesidade pode ser um primeiro passo na tentativa da melhora ou prevenção da diabetes tipo II. Pinheiro (14) defende que os portadores desta doença participem de um programa de auxilie na mudança de estilo de vida, onde seja amplamente abordado pontos como prática de exercícios físicos e mudanças de hábitos alimentares. O mesmo autor ressalta que estas mudanças auxiliam reduzir o risco do da diabetes tipo II, e no caso de já possuir a doença, o programa auxiliaria no controle da mesma promovendo qualidade de vida. Para pacientes com diabetes do tipo II realizar atividades físicas com controle da intensidade, duração e densidade (ou seja, exercício físico) seria o ideal para atingir os objetivos anteriormente (redução da massa gorda, melhora da sensibilidade a insulina e controle da glicemia). Alinhar esses objetivos com o sistema de saúde pública para combate ao sedentarismo e doenças que podem causar 
complicações e limitações aos indivíduos quando atingirem a fase adulta e $3^{\mathrm{a}}$ idade seria interessante. Isso poderia evitar o uso excessivo de remédios para o tratamento. A prática regular de exercício físico teria de ser um investimento a longo prazo, o que influenciaria todos os membros da família, para realizar atividades importantes do seu cotidiano quando assumirem uma idade avançada. Isso traria segurança para ter hábitos saudáveis e maior qualidade de vida. Essas questões vão além de aprendizado, pois estão relacionadas com a construção de uma identidade coerente a uma sociedade que queremos futuramente. O exercício físico se torna uma ferramenta aplicável para evitar e tratar a diabetes do tipo II.

\section{BENEFÍCIOS DA ATIVIDADE} FÍSICA E DO EXERCÍCIO FÍSICO NA SAÚDE DE PESSOAS COM DIABETES

Além de contribuir para o desenvolvimento estético e cognitivo, a atividade física estimula a produção de anticorpos para algumas doenças infeciosas, e além disso contribui pra prevenção e tratamento de outros tipos de doenças, como as crônicas não transmissíveis (i.e., a diabetes tipo II) (15). Segundo Nahas (16) a atividade física é qualquer movimento produzido pelo corpo humano que resulta em algum gasto energético. Ela deve ser praticada por todos, independente da idade e da condição física. O mesmo autor, ressalta que a prática de atividades físicas pode proporcionar diversos benefícios, como por exemplo, maior resistência a certas doenças, gasto de energia e manutenção da boa forma física, além de promover a diminuição do stress e aumento da autoestima. Azevedo (17) cita que a atividade física auxilia na manutenção da saúde do ser humano. Ela, além de fornecer estímulos para as capacidades físicas, pode também contribuir no controle e diminuição de doenças do coração e do sistema ósseo, como por exemplo a osteoporose. Para Bracco (18) a atividade física quando é praticada de forma assídua pode proporcionar benefícios para a saúde, no qual podem ser percebidos pelo indivíduo a longo, médio e curto prazo, como por exemplo, a capacidade de respiração, o controle de peso e o bem estar do indivíduos. Para o mesmo autor, quando o ser humano é considerado ativo desde a infância, ele possui mais chances de virar um adulto praticante de atividades físicas, podendo manter os níveis de desenvolvimento e saúde em uma taxa elevada, prevenindo assim doenças crônicas. Segundo o Ministério da Saúde (19) a prática regular de alguma atividade física deve ser considerada como algo fundamental para o controle da diabete e melhora na qualidade de vida, sendo possível melhorar o controle glicêmico, diminuir o risco de doenças coronárias a aumentar a captação de glicose no tecido muscular. A realização de atividades físicas regularmente pode acelerar o processo de 
adaptação hormonal e metabologia para o uso correto da insulina no corpo do paciente (20).

A prática de atividades físicas de qualquer natureza, pode proporcionar a um indivíduo portador da diabetes diversos benefícios, como por exemplo, auxílio para o controle da glicemia no sangue com melhora da sensibilidade da insulina. Este benefício de controle da glicemia pode ser entendido como imediato, ocorrendo no primeiro dia de treinamento. O benefício a longo prazo é, por exemplo, o aumento da ação da insulina no organismo. Este fenômeno é responsável por hipoglicemias que ocorrem até 48 horas após as atividades físicas, sendo explicado pela reposição de glicogênio pelas células e pelo gasto energético causado pela recuperação do organismo. Vicent et al. (21) aponta que a atividade física previne e combate as doenças do coração e a diabetes tipo II. Ele defende que a atividade física previne a falta de controle da glicemia, proporcionando um atraso no avanço do desequilíbrio glicêmico. Pesquisas realizadas pela American Diabetes Association (22) apontam que os pacientes que possuem diabetes do tipo II, quando são submetidos a algum tipo de atividade física com duração de 30 a 40 minutos por dia, tiveram suas funções das células betas maximizadas, ou seja, houve uma mudança significativa na sensibilidade de insulina. Segundo a mesma pesquisa, a mudança que as células pancreáticas recebem como resposta ao movimento praticado resultam na diminuição do mRNA para a pró-insulina e glicoquinase no pâncreas, ou seja, estes fatores auxiliam na diminuição da produção da insulina.

Koranyl et al. (23) informa que as atividades físicas melhor recomendadas para pessoas com diabetes são aquelas em que há a possibilidade de controlar a sua intensidade, na qual pode existir uma variação de energia gasta, levando em consideração o metabolismo e a limitação de cada indivíduo. Portanto, como há controle da intensidade, duração e de outros parâmetros relacionados ao esforço, esses movimentos passam a ser caracterizados como exercício físico. Para extrair os benefícios, além de alcançar/manter o peso corporal ideal é necessário que o exercício físico tenha gasto energético considerável (24). Exercícios que envolvem resistência muscular generalizada são eficazes na motivação do indivíduo com diabetes, o incentivando a continuar com as atividades. A musculação é um exercício físico considerado eficiente no combate a obesidade e o sobrepeso, podendo contribuir também na redução de doenças, como por exemplo a diabetes (21). Qualquer exercício físico praticado assiduamente com adequada intensidade apresenta aumento do gasto energético e metabolismo, consequentemente num armazenamento menor de gordura, melhorando o funcionamento das células e órgãos. 
A caminhada é uma atividade física comum na rotina da maioria dos seres humanos, e para os com diabetes pode ser proveitosa se realizada em intensidade controlada e por tempo adequado (2). Todavia, devido a possíveis complicações ou doenças, como por exemplo, a artrite/artrose, os indivíduos com diabetes podem realizar outras atividades que não exijam sustentação de todo o peso corporal, tais como, o ciclismo, a natação e hidroginástica (2). Desta forma, a prática de exercícios físicos pode prevenir a doenças e também tratar os pacientes diabéticos, podendo corroborar na melhora do aproveitamento da glicose no metabolismo, auxiliando ainda na diminuição dos maiores fatores de riscos que favorecem desenvolver a diabetes. Ou seja, o exercício físico atua como um dos principais protagonistas na prevenção e no tratamento da diabete mellitus para pacientes que não são insulinodependentes. E quando prescrito corretamente pode melhorar a qualidade de vida e o metabolismo dos pacientes.

A frequência da prática do exercício físico (entre 5 a 7 vezes por semana) é fundamental para que a sensibilidade a insulina possa aumentar e consequentemente assim, alcançar/ manter o peso ideal (25). O exercício físico melhora significativamente o metabolismo e controle hormonal dos indivíduos portadores da diabetes do tipo II, pois durante sua prática, existe a contração muscular, no qual assegura a translocação da GLUT4 no qual é uma proteína dependente da disponibilidade de insulina.
Quando ao treinamento com uso da força para pacientes diabéticos é prescrito, proporciona a este público o aumento do fluxo sanguíneo, aumento de massa muscular, aumento da captação da glicose e redução de gorduras circulantes. Os devidos cuidados para com este público, como a medida da glicemia pré, intra e pós-treino, devem ser realizados, além da hidratação, uma vez que este tipo de exercício pode influenciar na quantidade de glicose disponível, por solicitar essencialmente a via glicolítica (26). O exercício físico no qual utiliza a força como principal componente traz importância para o tratamento e prevenção da diabetes do tipo II, pois as adaptações fisiológicas como a hipertrofia muscular aumentam os sítios com possibilidade de captação da glicemia em excesso (26).

\section{CONSIDERAÇÕES FINAIS}

O exercício físico pode ser utilizado como forma de promover um estilo de vida saudável, sendo comprovado cientificamente como meio para favorecer uma população consciente e com melhor expectativa de vida, promovendo um futuro com maior qualidade de vida e menor frequência de doenças crônicas, como a diabetes tipo II. Maneiras de favorecer o controle e prevenção dessa doença foram relatadas. As tecnologias disponíveis que favorecem o sedentarismo podem ser convertidas para estimular maior quantidade de movimentos e promoção da saúde. 


\section{REFERÊNCIAS}

1. INSTITUTO BRASILEIRO DE GEOGRAFIA E ESTATISTICA. Práticas de esporte e atividades físicas Rio de Janeiro: IBGE; 2015 [Available from: https://biblioteca.ibge.gov.br/visualizacao/ livros/liv100364.pdf.

2. Goldstein BJ. Insulin resistance: from benign to type 2 diabetes mellitus. Rev Cardiovasc Med. 2003;4 Suppl 6:S3-10.

3. Wild S, Roglic G, Green A, Sicree R, King H. Global prevalence of diabetes: estimates for the year 2000 and projections for 2030. Diabetes Care. 2004;27(5):1047-53.

4. Ortiz MCA, Zanetti ML. Levantamento dos fatores de risco para diabetes mellitus tipo 2 em uma instituição de ensino superior. Revista Latino-Americana de Enfermagem. 2001;9:58-63.

5. Bastos J. Educação e tecnologia (DIGIT). Apostila apresentada na disciplina de Filosofia e História da Educação Tecnológica no Mestrado em Tecnologia do PPGTE do CEFET-PR. 1998.

6. Cerezo JAL. Ciência, tecnologia e sociedade: o estado da arte na Europa e nos Estados Unidos. In: Sasntos LW, editor. Ciencia, tecnologia e sociedade: o desafio da interação. Londrina: IAPAR; 2002. p. 3-39.

7. Previtale AP. A importância do brincar. Biblioteca Digital da UNICAMP: Universidade Estadual de Campinas; 2006.

8. Hanauer FJ. Impacto da Informática nas Relações Humanas

2005 [Available from: https://www.researchgate.net/ publication/242147036_Impacto_da_Informatica_nas_ Relacoes_Humanas.

9. Velloso MAL. Protegendo os inocentes. Diretoria da Prodemge. 2007.

10. Saúde. B-MdSDdGdEe. Política Nacional de Educação Permanente em Saúde In: Secretaria de Gestão do Trabalho e da Educação na Saúde DdGdEeS, editor. Brasília: Ministério da Saúde; 2009.

11. Matsudo SM, Matsudo VR, Araújo T, Andrade D, Andrade E, Oliveira L, et al. Nível de atividade física da população do Estado de São Paulo: análise de acordo com o gênero, idade, nível socioeconômico, distribuição geográfica e de conhecimento. Rev bras cienc mov. 2002;10(4):41-50.

12. Zlochevsky ERM. Obesidade na infância e adolescência. Rev paul pediatr. 1996;14(3):124-33.

13. ABESO. Estudos sobre obesidade na infância e adolescência revelam novas descobertas acerca do problema: ASSOCIAÇÃO BRASILEIRA, PARA O ESTUDO DA OBEŚIDADE E DA SÍNDROME METABÓLICA; 2011 [Available from: www.abeso.org. br.

14. Pinheiro M. Exercícios Para Diabetes: Benefícios E Como Evitar A Hipoglicemia 2020 [Available from: https://www.tuasaude.com/exerciciospara-diabetes/.

15. Marietto V. Atividade Física como Lazer. 2007. 16. Nahas MV. Atividade física, saúde e qualidade de vida: conceitos e sugestões para um estilo de vida ativo: Midiograf; 2001.

17. Júnior MRdA. Influência da atividade física na adolescência sobre o nível de atividade física na idade adulta. Pelotas: Universidade Federal de Pelotas; 2004.

18. Pediatria SBd, Nutrologia DCd. Atividade física na infância e na adolescência: guia prático para o pediatra. SBP Rio de Janeiro; 2008.

19. BRASIL. Estratégias para o cuidado da pessoa com doença crônica: diabetes mellitus. In: Saúde MdSDdGdEe, editor. Brasília: Ministério da Saúde; 2013.

20. de Lara FN. O efeito agudo do exercÃcio de for $\tilde{A} \int$ ae da caminhada, na glicemia de um indivÃduo sedent $\tilde{A}_{i}$ rio, diab $\tilde{A}(\mathrm{C}$ tico do tipo 2. RBPFEX-Revista Brasileira de Prescri $\tilde{A} \int \tilde{A} \mathcal{E}$ oe Fisiologia do Exerc Ãcio. 2009;3(15).

21. Vincent SD, Pangrazi RP, Raustorp A, Tomson LM, Cuddihy TF. Activity levels and body mass index of children in the United States, Sweden, and Australia. Med Sci Sports Exerc. 2003;35(8):1367-73.

22. American Diabetes Association. Standards of medical care in diabetes 2019. Diabetes Care. 2019;42(Suppl 1):S124-S38.

23. Koranyi K, Rasnake LK, Tarnowski KJ. Nursing bottle weaning and prevention of dental caries: a survey of pediatricians. Pediatr Dent. 1991;13(1):32-4.

24. Dornas WC, de Oliveira TT, Nagem TJ. Exercício físico e diabetes mellitus tipo 2. Arquivos de Ciências da Saúde da UNIPAR. 2011;15(1).

25. Fernandes CAM, Junior NN, Tasca RS, Pelloso SM, Cuman RKN. A importância da associação de dieta e de atividade física na prevenção e controle do Diabetes mellitus tipo 2. Acta Scientiarum Health Sciences. 2005;27(2):195-205.

26. Júnior CAS, de Almeida RS, Baggio T, de Oliveira Assumpçao C. Efeito do treinamento de força em portadores de Diabetes Mellitus tipo II. Revista Brasileira de Prescrição e Fisiologia do Exercício (RBPFEX). 2014;8(47):12.

OBSERVAÇÃO: Os autores declaram não existir conflitos de interesse de qualquer natureza. 